\title{
TRATAMIENTO DE FISIOTERAPIA MULTIMODAL BASADO EN UN PARADIGMA BIOCONDUCTUAL EN EL ABORDAJE DE MIGRAÑA CRÓNICA: ESTUDIO DE UN CASO.
}

\author{
Luis Suso-Martí, MSc ${ }^{1,2}$; Swann Atger, FT ${ }^{1}$; Ferran Cuenca-Martínez, MSc ${ }^{1,2}$. \\ 1. AGClinic Fisioterapia Avanzada. Madrid (España). \\ 2. Grupo de Investigación Motion in Brains, Instituto de Neurociencia y Ciencias del Movimiento (INCIMOV), \\ Centro Superior de Estudios Universitarios La Salle, Universidad Autónoma de Madrid, España.
}

\begin{abstract}
Correspondencia:
Ferran Cuenca-Martínez, PT, MSc. Grupo de Investigación INCIMOV, CSEU La Salle. Universidad Autónoma of Madrid. Calle La Salle, $n^{\circ} 10,28023$ Madrid, España Teléfono: (+34) 962277265 E-Mail: fecuen2@gmail.com

\section{Conflicto de Intereses:}

Los autores del presente manuscrito declaran no presentar ningún conflicto de interés y además el presente estudio no ha sido presentado en ningún evento científico.
\end{abstract}

Financiación

Los autores declaran no haber recibido financiación/compensación para el desarrollo de esta investigación.

DOI:

https://doi.org/10.37382/jomts.v1i1.11

Recepción del Manuscrito:

8-Enero-2019

Aceptación del Manuscrito:

11- Мayo-2019

Licensed under: CC BY-NC-SA 4.0

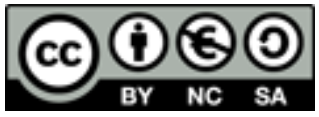

\section{RESUMEN}

La migraña crónica es una cefalea primaria en la que se producen más de 15 episodios de cefalea al mes. Esta patología es la séptima causa de discapacidad en el mundo, y la literatura científica actual sugiere que un tratamiento multimodal desde un paradigma bioconductual se podría precisar para esta población.

Una mujer de 36 años diagnosticada de migraña crónica que presentó cefaleas 30 días al mes y altos niveles de discapacidad. Se evaluaron mediante autoinforme kinesiofobia, pensamientos catastróficos, impacto del dolor de cabeza, discapacidad por migraña y niveles de ansiedad y depresión. Como variables motoras, la resistencia de los flexores profundos y finalmente se registró la frecuencia de dolor.

Se tomaron cinco mediciones durante un total de 120 días.

Doce sesiones de tratamiento incluyendo terapia manual neuro-ortopédica, ejercicio terapéutico y estrategias bioconductuales, incluyendo educación terapéutica en dolor fueron incluidos.

El tratamiento multimodal basado en un paradigma multimodal produjo una reducción sustancial en la frecuencia de las cefaleas, la discapacidad, el catastrofismo y el impacto de la migraña crónica en la vida de la paciente.

Palabras Clave: Migraña crónica, fisioterapia bioconductual, terapia manual neuroortopédica, ejercicio terapéutico, educación terapéutica. 


\section{INTRODUCCIÓN}

La migraña es una enfermedad neurológica considerada como una entidad clínica compleja, donde la interacción de factores biológicos, psicológicos y del entorno juegan un papel crítico en la aparición del dolor (Carod-Artal et al., 2012).

De acuerdo con la Asociación Internacional de Cefaleas, la migraña crónica es definida cómo una cefalea primaria dónde se producen más de 15 episodios al mes (Headache Classification Committee of the International Headache Society (IHS), 2013). En relación al dolor asociado a la migraña crónica, se ha sugerido que distintas variables psicosociales, tales como el miedo-evitación, los niveles de ansiedad, el estrés, los pensamientos, las creencias de autoeficacia así como otros factores tanto afectivo-emocionales como cognitivos van a influir en la experiencia dolorosa $\mathrm{y}$ en consecuencia en la conducta $\mathrm{y}$ afrontamiento ante la misma (Burstein et al., 2015).

El tratamiento de primera opción en estos pacientes es el farmacológico. Sin embargo, las investigaciones acerca del papel de la sensibilización central y de la hiperexcitabilidad del complejo trigémino-cervical así como de la influencia de factores psicosociales en el dolor de los pacientes, ponen de manifiesto la necesidad de buscar nuevas alternativas en el tratamiento de los pacientes con migraña crónica, evitando además del abuso de medicación y el aumento de los síntomas tras el uso prologando de los mismos (Bartsch \& Goadsby, 2003; Negro \& Martelletti, 2011).

\section{PRESENTACIÓN DEL CASO}

La paciente es una mujer de 36 años $(56 \mathrm{Kg}, 1.68 \mathrm{~m})$ que acude a consulta de Fisioterapia con diagnóstico de migraña. Los episodios de dolor de cabeza comenzaron en 2002, estando en seguimiento por el neurólogo desde entonces, que le ha prescrito un conjunto de medidas farmacológicas tales como Botox (250 Ui), Vimpat, Pregabalina o Tryptizol, sin éxito terapéutico. Se le realizó una exploración neurológica en la que no se obtuvieron hallazgos relevantes, siendo diagnosticada de migraña crónica refractaria
Actualmente se administra de forma intradérmica Inmigran con una reducción parcial del dolor en momentos de crisis. Como antecedentes familiares, tanto su abuela, padre y madre tienen migraña crónica, y esta última también está diagnosticada por fibromialgia.

\section{Hallazgos clínicos}

En la exploración de Fisioterapia, se evaluó el rango de movimiento cervical, encontrándose valores ligeramente disminuidos en todos los planos de movimiento acompañado de la presencia de dolor. Se realizó también una valoración de la movilidad accesoria cervical, encontrándose rigidez y dolor en la exploración del movimiento postero-anterior de C2C7, además también de espasmo de la musculatura cervical con presencia de puntos gatillo miofasciales en músculos trapecio superior, suboccipitales, escalenos y esternocleidomastoideo.

Posteriormente, se realizó una valoración neurodinámica, utilizando los test neurodinámicos ULNT1, ULNT2B, ULNT3, test de puesta en tensión de la rama mandibular del trigémino, Slump test y test de flexión pasiva modificada. En todos los casos se encontró una respuesta de mecanosensibilidad aumentada, dolor y reproducción de los síntomas del paciente tanto a nivel de miembro superior como a nivel cráneo-facial. Además, se realizó el test de resistencia de los flexores profundos (TRFP), para evaluar la resistencia isométrica contra la gravedad de la musculatura profunda del cuello. El resultado de este test está reflejado en los segundos en los que el paciente puede mantener la flexión cráneo-cervical (Harris et al., 2005).

Se aplicaron, además, medidas de autoinforme para evaluar diferentes aspectos psicosociales, el impacto del dolor de cabeza y su discapacidad asociada. Los resultados de las medidas están descritos en la Tabla 1.

Los pensamientos catastróficos ante el dolor fueron evaluados mediante la escala validada al español de catastrofismo ante el dolor (PCS). Es una escala autoadministrada que presenta adecuadas propiedades psicométricas y consta de 13 ítems que comprenden 3 
Tabla 1. Mediciones de los hallazgos clínicos.

\begin{tabular}{|c|c|c|c|c|c|}
\hline MEDICIÓN & 0 & 1 & 2 & 3 & 4 \\
\hline Tiempo & Pre-INT & 30 días & 60 días & 90 días & 120 días (post-INT) \\
\hline PCS & 33 & 30 & 22 & 20 & 20 \\
\hline TSK & 17 & 17 & 15 & 15 & 15 \\
\hline HIT-6 & 68 & 62 & 53 & 49 & 47 \\
\hline MIDAS & 53 & 40 & 13 & 8 & 8 \\
\hline HADS & $11 / 4$ & $10 / 4$ & $8 / 3$ & $6 / 2$ & $6 / 2$ \\
\hline TRFP (seg.) & 11 & 15 & 23 & 30 & 34 \\
\hline Frecuencia de dolor por mes & 30 & 27 & 19 & 10 & 4 \\
\hline
\end{tabular}

(días)

INT: intervención, seg: segundos, PCS: escala de catastrofismo ante el dolor, TSK: escala tampa de kinesiofobia, HIT-6: cuestionario para valorar el impacto del dolor de cabeza y discapacidad asociada, MIDAS: cuestionario de discapacidad por migraña, HADS: escala de ansiedad y depresión y TRPF: test de resistencia de los flexores profundos.

dimensiones: a) rumiación; b) magnificación, y c) desesperanza (García Campayo et al., 2008).

Los niveles de kinesiofobia fueron registrados mediante la escala tampa de kinesiofobia validada al español (TSK-11). Este instrumento presenta adecuadas propiedades psicométricas y la puntuación total oscila entre 11 y 44 puntos, siendo las puntuaciones más altas las que indican mayores niveles de kinesiofobia (Gómez-Pérez et al., 2011).

Para valorar el impacto del dolor de cabeza y la discapacidad asociada se utilizó el Headache Impact Test (HIT-6). La versión española de este cuestionario consiste en un cuestionario auto-administrado de seis ítems que mide el impacto y la relevancia del dolor de cabeza en la vida del paciente (Gandek et al., 2003).

Los niveles de discapacidad por migraña fueron evaluados mediante el cuestionario de evaluación de discapacidad por migraña (MIDAS) que mide la discapacidad en las actividades de la vida diaria relacionada con la cefalea en base a cinco preguntas. La puntuación se obtiene de la suma de los días perdidos por cefalea (Fernández-Concepción \& Canuet-Delis., 2003).

El estado de ansiedad y depresión se valoró con la Escala de Ansiedad y Depresión (HADS). Esta escala se trata de un instrumento auto administrado que contiene 14 ítems subdivididos en dos subescalas, una para la ansiedad y otra para depresión demostrado que presenta adecuadas propiedades psicométricas (Quintana et al., 2003). Los resultados reflejan un problema clínico cuando se encuentran valores superiores a 11 para cualquiera de las dos subescalas.

\section{Intervención}

La paciente recibió un total de 12 sesiones de tratamiento divididas en tres meses, a razón de una sesión por semana de una duración aproximada de 50 minutos. El resumen del tratamiento está especificado en la Tabla 2.

\section{RESULTADOS}

Cinco mediciones fueron tomadas en el presente estudio: la primera fue pre-intervención, de la segunda a la cuarta medida se realizaron una cada mes durante la duración del tratamiento y finalmente, la última medición se tomó un mes después de finalizar la intervención (Figura 1). Los resultados están descritos en la Tabla 1. 
Figura 1. Cronograma de las mediciones y la intervención.

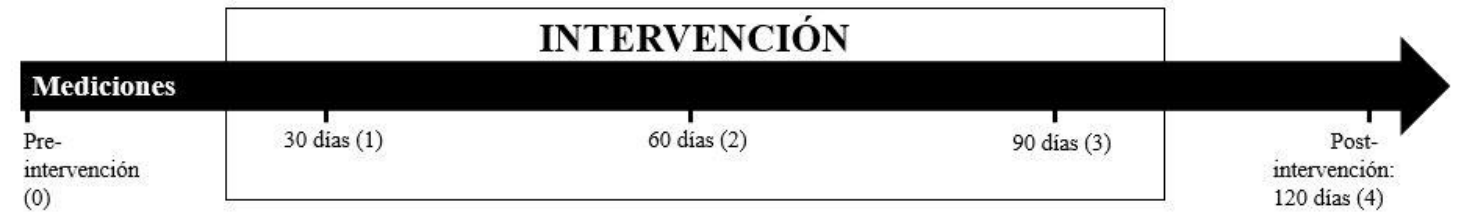

\section{DISCUSIÓN}

En este informe de un caso, se describe la evaluación y el tratamiento de una paciente con migraña basado en un abordaje bioconductual desde un tratamiento multimodal de fisioterapia.

En la literatura científica actual, no existe evidencia acerca del tratamiento multimodal basado en un paradigma bioconductual para una intervención en pacientes con migraña crónica. Sin embargo, KindelanCalvo et al., (2014) realizaron una revisión sistemática donde se observó que las intervenciones con educación terapéutica de forma aislada sí que han mostrado evidencia, en primer lugar moderada en la mejora de la calidad de vida y, en segundo lugar, evidencia fuerte en la disminución de la discapacidad y de la frecuencia de dolor en pacientes adultos con migraña crónica a medio plazo. Además, Holroyd et al., (2010) realizaron un ensayo clínico en el que se encontró que añadir un tratamiento bioconductual centrado en las estrategias de afrontamiento al dolor a un tratamiento farmacológico fue más efectivo que este último de forma aislada.

Respecto a la terapia manual neuro-ortopédica utilizada, en base a las relaciones neurofisiológicas entre la columna cervical y el complejo trigeminal, se propusieron técnicas hipoalgésicas articulares en los niveles cervicales altos para provocar una proceso de modulación del complejo trigémino-cervical (La Touche et al., 2013).

Existe una carencia de literatura científica de buena calidad metodológica acerca de las técnicas de terapia manual neuro-ortopédica utilizadas en el tratamiento de estos pacientes, aunque parece que el uso de técnicas clásicas tales como el masaje presentan efectos similares a los conseguidos mediante estrategias de relajación, y además, se desaconseja el uso de la técnica de manipulación cervical (Chaibi et al., 2011; Posadzki \& Ernst, 2011).

El proceso de sensibilización del núcleo trigéminocervical es considerado actualmente como uno de los posibles mecanismos pato-biológicos presentes en la migraña crónica (Bartsch \& Goadsby, 2003). En este aspecto, la movilización neurodinámica ha mostrado ser capaz de modificar la expresión de los canales iónicos y de lograr una posible modulación de la hiperexcitabilidad nerviosa, con lo que se sugiere, por tanto, que puede ser una herramienta de gran potencial terapéutico para los pacientes con migraña crónica (Santos et al., 2012; Gilbert et al., 2015).

Por otro lado, a pesar de la escasa literatura científica respecto al papel del ejercicio en la migraña crónica basado en un paradigma bioconductual, se utilizó en el presente estudio un programa de ejercicio terapéutico enfatizando en el ejercicio como estrategia de afrontamiento activo ante el dolor. El uso del ejercicio terapéutico en los pacientes con migraña crónica es considerado un tratamiento potencialmente beneficioso tanto por la modulación neuro-bioquímica producida por el mismo, como por la disminución de la influencia de los factores psicológicos implicados en esta entidad clínica (Naugle et al., 2012; Koseoglu et al., 2015; Irby et al., 2016). Además, uno de los hallazgos encontrados en la exploración clínica fue la reducción de la fuerza-resistencia de la musculatura flexora profunda cráneo-cervical. Estos datos concuerdan con los hallados por Florencio et al., (2016), con lo que parece necesario la inclusión de un programa de ejercicios que vaya enfocado a mejorar la fuerza-resistencia de esta musculatura del plano profundo cráneo-cervical así como del control neurosensoriomotor. 
Tabla 2: Descripción de la intervención.

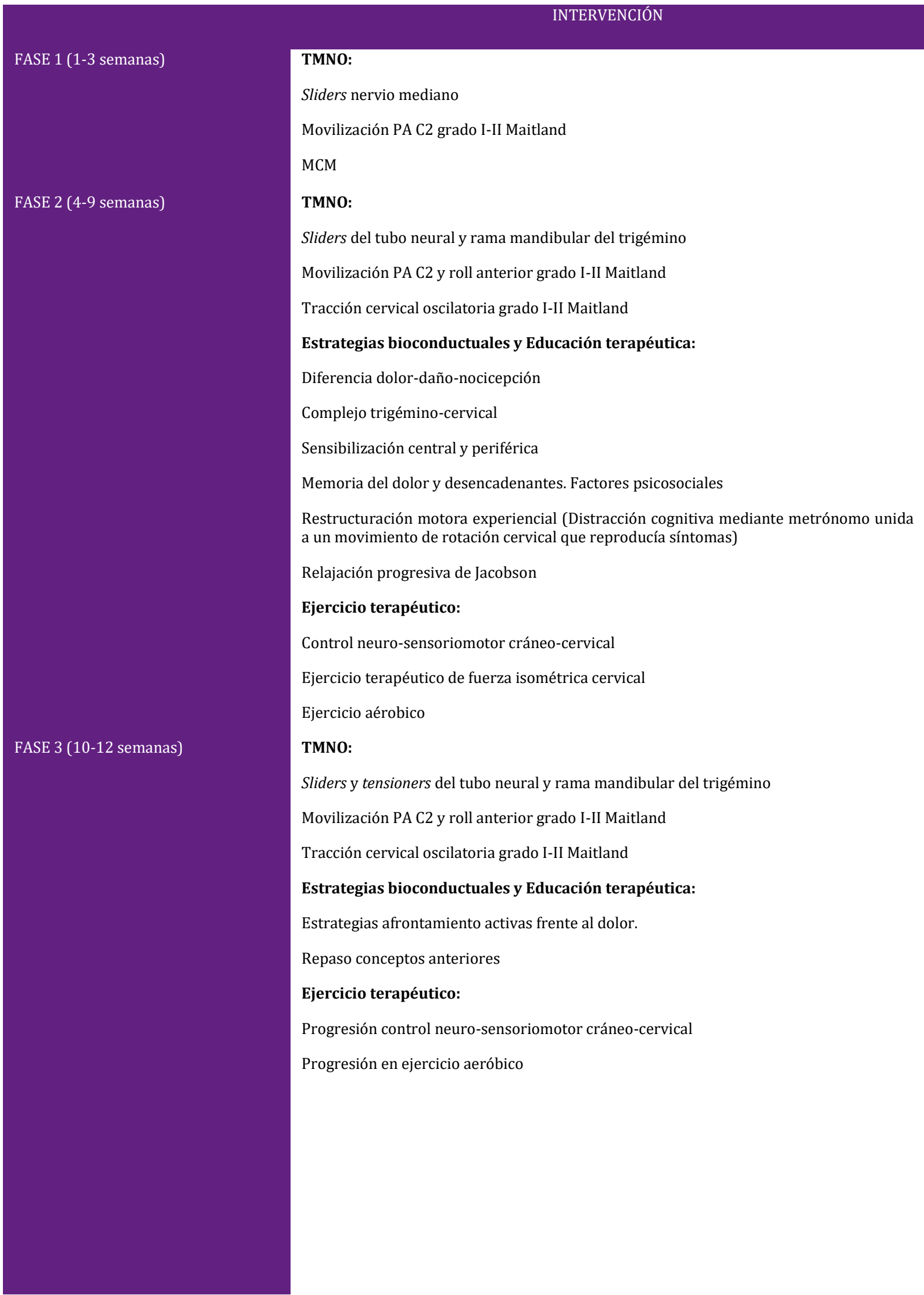

TMNO: Terapia Manual Neuro-Ortopédica, MCM: Movilización Con Movimiento, PA: Postero-Anterior. 
El tratamiento multimodal basado en un paradigma bioconductual ha mostrado resultados similares en otras entidades clínicas. Engquist et al., (2013) encontraron que un tratamiento multimodal basado en un abordaje bioconductual centrado en el aumento de las estrategias de afrontamiento activas, el manejo del estrés y un programa de ejercicio terapéutico tanto general como específico cervical obtuvo resultados similares a los obtenidos mediante cirugía de retirada del disco intervertebral y de osteofitos junto con la fusión vertebral además del mismo programa de Fisioterapia multimodal en pacientes con radiculopatía cervical crónica a los dos años.

Por otro lado, Beltran-Alacreu et al., (2015) obtuvieron resultados beneficiosos en la disminución de la intensidad de dolor, mejora de la discapacidad y en la influencia de variables psicosociales mediante un tratamiento de Fisioterapia multimodal bajo un prisma bioconductual en el estudio de un paciente con cefalea de tipo tensional crónica.

Los resultados de este estudio y los hallados en la literatura científica en entidades clínicas similares muestran resultados positivos mediante una intervención multimodal basada en un paradigma bioconductual en pacientes con dolor crónico. En este caso, además este tratamiento mostró mejores resultados que los tratamientos recibidos previos a la intervención descrita. La hipótesis de los autores de este estudio es que la gran relevancia de los factores psicosociales presentes en la entidad clínica a podido conllevar que el abordaje desde un punto bioconductual y la adquisición de estrategias de afrontamiento activas frente al dolor mediante el ejercicio hayan podido tener un papel crítico en el éxito terapéutico.

\section{Limitaciones}

Existen varias limitaciones a tener en cuenta en el presente estudio de un caso. En primer lugar, no se realizó un seguimiento a largo plazo, por lo que el resultado de este tratamiento solo puede ser tenido en cuenta a corto-medio plazo. En segundo lugar, tampoco conocemos los efectos ni la dosificación de la medicación por parte de la paciente y sus interacciones con los resultados obtenidos, aunque reportó que esta fue disminuyendo progresivamente. En tercer lugar, el estudio del estrés en los pacientes con migraña crónica es de reciente actualidad, se podría haber registrado la actividad del sistema nervioso simpático-excitatorio mediante variables mono-inervadas como la conductancia de la piel o por el contrario, variables de registro diario como la variabilidad de la frecuencia cardíaca para podernos ofrecer mayor información neurofisiológica. En cuarto lugar, el hecho de ser una intervención multimodal no permite conocer en qué grado afecta cada componente del tratamiento a los resultados obtenidos, y por tanto la efectividad de cada tipo de abordaje en el tratamiento de la MC. Por último, otra limitación es la falta de registro de variables somatosensoriales tales como la sumación temporal, la algometría o la discriminación de dos puntos debido a que los pacientes con migraña crónica han mostrado diferentes alteraciones somatosensoriales (Weissman-Fogel et al., 2003).

\section{CONCLUSIÓN}

Un tratamiento de Fisioterapia multimodal basado en un paradigma bioconductual mostró mejoras en la discapacidad por migraña y en la frecuencia de episodios de migraña al mes. Además, se encontraron mejoras relevantes en la influencia de variables psicosociales, así como de mejoras en la fuerzaresistencia de los músculos flexores profundos cráneocervicales. Es necesario realizar ensayos clínicos aleatorizados, bien diseñados y con un seguimiento a largo plazo para evaluar la efectividad de un tratamiento de Fisioterapia multimodal, basado en un paradigma bioconductual, para los pacientes con migraña crónica para poder extrapolar conclusiones más sólidas sobre el papel de la Fisioterapia en la migraña crónica. 


\section{FRASES DESTACADAS}

$>$ Paciente diagnosticada de Migraña Crónica con cefalea 30 días al mes y alto nivel de discapacidad.

$>$ Se realizaron doce sesiones de tratamiento multimodal basado en un paradigma bioconductual.

$>$ Se produjo una reducción de la discapacidad, la frecuencia de las cefaleas y de su impacto.

\section{REFERENCIAS}

Bartsch T, Goadsby PJ. The trigeminocervical complex and migraine: current concepts and synthesis. Curr Pain Headache Rep. 2003;7(5):371-6.

Beltran-Alacreu H, Lopez-de-Uralde-Villanueva I, La Touche R. Multimodal Physiotherapy Based on a Biobehavioral Approach as a Treatment for Chronic Tension-Type Headache: A Case Report. Anesthesiol Pain Med. 2015;5(6): e32697. DOI: http://dx.doi.org/10.5812/aapm.32697.

Burstein R, Noseda R, Borsook D. Migraine: multiple processes, complex pathophysiology. $J$ Neurosci. Society for Neuroscience. $\quad 2015 ; 35(17): 6619-29 . \quad$ DOI: http://dx.doi.org/10.1523/JNEUROSCI.0373-15.2015.

Carod-Artal FJ, Irimia P, Ezpeleta D. Chronic migraine: definition, epidemiology, risk factors and treatment. Rev Neurol. 2012;54(10):629-37.

Chaibi A, Tuchin PJ, Russell MB. Manual therapies for migraine: a systematic review. J Headache Pain. 2011;12(2):127-33. DOI: http://dx.doi.org/10.1007/s10194-011-0296-6.

Engquist M, Löfgren H, Öberg B, Holtz A, Peolsson A, Söderlund A, Vavruch L, Lind B. Surgery versus nonsurgical treatment of cervical radiculopathy: a prospective, randomized study comparing surgery plus physiotherapy with physiotherapy alone with a 2-year follow-up. Spine (Phila Pa 1976). 2013;38(20):1715-22

DOI: http://dx.doi.org/10.1097/BRS.0b013e31829ff095.

Fernández-Concepción O, Canuet-Delis L. Disability and quality of life in patients with migraine: determining factors. Rev Neurol. 36(12):1105-12.

Florencio LL, Oliveira AS, Lemos TW, Carvalho GF, Dach F, Bigal ME, Falla D, Fernández-de-las-Peñas C, BevilaquaGrossi D. Patients with chronic, but not episodic, migraine display altered activity of their neck extensor muscles. $J$ Electromyogr Kinesiol. 2016;30:66-72.

Gandek B, Alacoque J, Uzun V, Andrew-Hobbs M, Davis K. Translating the Short-Form Headache Impact Test (HIT-6) in 27 countries: Methodological and conceptual issues. Qual Life Res. 2003;12(8):975-9 DOI: http://dx.doi.org/10.1023/A:1026171315263.
García Campayo J, Rodero B, Alda M, Sobradiel N, Montero J, Moreno S. Validation of the Spanish version of the Pain Catastrophizing Scale in fibromyalgia. Med clínica. 2008;131(13):487-92.

Gilbert KK, Roger James C, Apte G, Brown C, Sizer PS, Brismée J-M, Smith MP. Effects of simulated neural mobilization on fluid movement in cadaveric peripheral nerve sections: implications for the treatment of neuropathic pain and dysfunction. J Man Manip Ther. 2015;23(4):219-25 DOI: http://dx.doi.org/10.1179/2042618614Y.0000000094.

Gómez-Pérez L, López-Martínez AE, Ruiz-Párraga GT. Psychometric Properties of the Spanish Version of the Tampa Scale for Kinesiophobia (TSK). J Pain. 2011;12(4):425-35 DOI: http://dx.doi.org/10.1016/j.jpain.2010.08.004.

Harris KD, Heer DM, Roy TC, Santos DM, Whitman JM, Wainner RS. Reliability of a measurement of neck flexor muscle endurance. Phys Ther. 2005;85(12):1349-55.

Headache Classification Committee of the International Headache Society (IHS). The International Classification of Headache Disorders, 3rd edition (beta version). Cephalalgia. 2013;33(9):629-808 DOI: http://dx.doi.org/10.1177/0333102413485658.

Holroyd KA, Cottrell CK, O’Donnell FJ, Cordingley GE, Drew JB, Carlson BW, Himawan L. Effect of preventive (beta blocker) treatment, behavioural migraine management, or their combination on outcomes of optimised acute treatment in frequent migraine: randomised controlled trial. $B M J$. 2010;341:c4871 DOI: http://dx.doi.org/10.1136/BMJ.C4871.

Irby MB, Bond DS, Lipton RB, Nicklas B, Houle TT, Penzien DB. Aerobic Exercise for Reducing Migraine Burden: Mechanisms, Markers, and Models of Change Processes. Headache J Head Face Pain. 2016;56(2):357-69 DOI: http://dx.doi.org/10.1111/head.12738.

Kindelan-Calvo P, Gil-Martínez A, Paris-Alemany A, PardoMontero J, Muñoz-García D, Angulo-Díaz-Parreño S, La Touche R. Effectiveness of Therapeutic Patient Education for Adults with Migraine. A Systematic Review and MetaAnalysis of Randomized Controlled Trials. Pain Med. 2014;15(9):1619-36 DOI: http://dx.doi.org/10.1111/pme.12505.

Koseoglu E, Yetkin MF, Ugur F, Bilgen M. The role of exercise in migraine treatment. $J$ Sports Med Phys Fitness. 2015;55(9):1029-36.

Naugle KM, Fillingim RB, Riley JL, III. A meta-analytic review of the hypoalgesic effects of exercise. $J$ Pain. 2012;13(12):1139-50 DOI: http://dx.doi.org/10.1016/j.jpain.2012.09.006.

Negro A, Martelletti P. Chronic migraine plus medication overuse headache: two entities or not? $J$ Headache Pain. 2011;12(6):593-601 DOI: http://dx.doi.org/10.1007/s10194011-0388-3.

Posadzki P, Ernst E. Spinal manipulations for the treatment of migraine: A systematic review of randomized clinical trials. Cephalalgia. 2011;31(8):964-70 DOI: http://dx.doi.org/10.1177/0333102411405226. 
Quintana JM, Padierna A, Esteban C, Arostegui I, Bilbao A, Ruiz I. Evaluation of the psychometric characteristics of the Spanish version of the Hospital Anxiety and Depression Scale. Acta Psychiatr Scand. 2003;107(3):216-21 DOI: http://dx.doi.org/10.1034/j.1600-0447.2003.00062.x.

Santos FM, Silva JT, Giardini AC, Rocha PA, Achermann AP, Alves AS, Britto LR, Chacur M. Neural Mobilization Reverses Behavioral and Cellular Changes That Characterize Neuropathic Pain in Rats. Mol Pain. 2012;8:57 DOI: http://dx.doi.org/10.1186/1744-8069-8-57.
La Touche R, París-Alemany A, Mannheimer JS, Angulo-DíazParreño S, Bishop MD, Lopéz-Valverde-Centeno A, von Piekartz H, Fernández-Carnero J. Does Mobilization of the Upper Cervical Spine Affect Pain Sensitivity and Autonomic Nervous System Function in Patients With Cervicocraniofacial Pain? Clin J Pain. 2013;29(3):205-15 DOI: http://dx.doi.org/10.1097/AJP.0b013e318250f3cd.

Weissman-Fogel I, Sprecher E, Granovsky Y, Yarnitsky D. Repeated noxious stimulation of the skin enhances cutaneous pain perception of migraine patients in-between attacks: clinical evidence for continuous sub-threshold increase in membrane excitability of central trigeminovascular neurons. Pain. 2003;104(3):693-700. 